\title{
Value of the CD8-CD3 Ratio for the Diagnosis of Mycosis Fungoides
}

Nicolas Ortonne, M.D., Nesimi Buyukbabani, M.D., Marie-Hélène Delfau-Larue, M.D., Ph.D., Martine Bagot, M.D., Ph.D., Janine Wechsler, M.D.

Departments of Pathology (NO, JW), Immunology (M-H D-L), and Dermatology (MB), Hôpital Henri Mondor, Créteil, France; and Department of Pathology (NB), Tip Fakültesi Patoloji Anabilim Dali Capa, Istanbul, Turkey

Histopathological diagnosis of mycosis fungoides is difficult, especially in early lesions that may be indistinguishable from inflammatory dermatoses. Mycosis fungoides is a clonal proliferation of mature epidermotropic CD4+ lymphocytes. The aim of this study was to determine the contribution of the CD8-CD3 ratio to the diagnosis of mycosis fungoides. We retrospectively compared the immunophenotypic characteristics of $\mathbf{3 0}$ mycosis fungoides with 28 inflammatory dermatoses. The diagnosis of mycosis fungoides was reinforced in all cases by the presence of a cutaneous dominant T-cell clonal population. To analyze exclusively the lymphocytic infiltrates, CD4, which is also expressed by histiocytes, was not considered. The CD8-CD3 ratio was determined separately in the epidermis and the dermis using two methods, one quantitative and the other semiquantitative. Concordance rates between the two methods were higher in epidermal than dermal infiltrates. The mean CD8-CD3 ratio was significantly lower for mycosis fungoides than control cases, with the difference being greater in the epidermal than the dermal component. Although not absolutely specific, a low $\mathrm{CD8}-\mathrm{CD} 3$ ratio in the epidermal component of a lymphocytic infiltrate supports the diagnosis of mycosis fungoides. It can be evaluated in routine practice using a semiquantitative approach.

KEY WORDS: CD3, CD8, Lymphocytes, Mycosis fungoides.

Mod Pathol 2003;16(9):857-862

Copyright $\odot 2003$ by The United States and Canadian Academy of Pathology, Inc.

VOL. 16, NO. 9, P. 857, 2003 Printed in the U.S.A.

Date of acceptance: May 16, 2003.

Address reprint requests to: Janine Wechsler, M.D., Département de Pathologie, hôpital Henri Mondor, 51 avenue du Maréchal de Lattre de Tassigny, 94010 Créteil, France; fax: $\begin{array}{llllllll}33 & 1 & 49 & 81 & 27 & 33 \text {; e-mail: }\end{array}$ janine.wechsler@hmn.ap-hop-paris.fr.

DOI: 10.1097/01.MP.0000084112.81779.BB
Mycosis fungoides is the most common subtype of primary cutaneous T-cell lymphomas (1). Clinically, the disease has a slow course with three distinct stages: patches, plaques, and tumors. Histologically, mycosis fungoides is characterized by a subepidermal bandlike infiltrate of lymphocytes associated with epidermotropism. Recent studies focused on the diagnostic value of histopathological parameters for the diagnosis of mycosis fungoides. However, mycosis fungoides is difficult to diagnose, and, especially in early lesions, distinction between mycosis fungoides and inflammatory diseases is sometimes impossible. It has been shown that the infiltrating lymphocytes in mycosis fungoides are predominantly $\mathrm{CD} 4+$, with a minority of $\mathrm{CD} 8+\mathrm{T}$ cells, but CD4 is also expressed by histiocytes, which can be plentiful in this context. Therefore, the aim of this study was to determine whether the CD8-CD3 ratio could be a marker for the diagnosis of mycosis fungoides. To address this issue, we retrospectively conducted quantitative and semiquantitative analyses of CD8-CD3 ratios in skin biopsies of 30 cases of early and plaque-stage mycosis fungoides and compared them with 28 inflammatory dermatoses. Diagnosis of mycosis fungoides was supported in all cases by the presence of a cutaneous clonal $\mathrm{T}$-cell receptor rearrangement.

\section{MATERIALS AND METHODS}

\section{Patients}

Two groups of patients were retrospectively constituted and studied.

The 30 mycosis fungoides patients fulfilled the following criteria: (1) presentation of multiple patches or plaques, or erythroderma, with strong clinical suspicion of mycosis fungoides; (2) presence of a T-cell receptor rearrangement in a frozen skin specimen; (3) absence of a circulating T-cell clone and circulating Sézary cells; (4) no evidence of extracutaneous disease at the time of diagnosis, 
(5) negative HIV 1 and 2 and HTLV tests. Patients included 24 men and 6 women (sex ratio: 4), with a median age of 50 years (range: 18-78 y). Twenty-four $(83 \%)$ patients had erythematous patches or erythematosquamous plaques; 2 had erythroderma. Three patients had atypical lesions: one had eczematous lesions; one had digitate plaques on the trunk, and one presented with follicular hyperkeratotic patches on the trunk and the extremities and had hyperkeratotic thickening of the palms and soles. Among the 28 cases with complete clinical information, median duration of the lesions before diagnosis was 7 years (range: 1-35 y). Seventeen $(60 \%)$ patients had received no treatment before diagnosis. Eight had received local corticotherapy; 3 had undergone phototherapy with UVA and psoralen, associated with local corticotherapy; and 1 had undergone radiation therapy.

The control group included 28 patients with inflammatory dermatoses: 10 drug-induced eruptions, 9 lichen planus, and 9 contact dermatitis.

\section{Histopathology and Immunohistochemistry}

One or two formalin-fixed biopsies from each patient were available. Five mycosis fungoides patients had undergone two sequential biopsies. Thus, 35 biopsies were analyzed in the mycosis fungoides group. Skin specimens from mycosis fungoides patients and controls were routinely processed, embedded in paraffin, and stained with hematoxylin-eosin. Paraffin sections were immunohistochemically labeled with the avidin-biotinperoxidase method using monoclonal antibodies directed against CD3 and CD8 antigens (DAKO, Glostrup, Denmark).

Mycosis fungoides infiltrates were morphologically categorized into three groups, as previously described (2). All slides were independently analyzed by two observers. Infiltrates were nonspecific, suggestive of mycosis fungoides, or typical of mycosis fungoides. Samples classified as typical were those with the characteristic histological features of mycosis fungoides: bandlike subepidermal infiltrate, presence of Sézary cells, single-cell epidermotropism, and/or clusters of cells forming Pautrier's microabscesses. Suggestive lesions were defined as slight-to-moderate lymphocytic infiltrates showing a linear distribution beneath the epidermis, presence of few Sézary cells, and focal single-cell epidermotropism, localized in the germinal layer of the epidermis. Lesions were considered nonspecific when patchy perivascular infiltrates were present in the absence of Sézary cells and lack of epidermotropism.

\section{Evaluation of the CD8-CD3 Ratio}

CD8-CD3 ratios were evaluated separately for the epidermis and the dermis, using two distinct methods. In the quantitative method, we counted CD3+ and CD8+ cells in the epidermis and the dermis and then calculated the corresponding CD8-CD3 ratios. Labeled epidermal cells were counted under high-power ( $40 \times$ objective) along the entire epidermis, which was $\geq 2 \mathrm{~mm}$ long in each sample. In the count of positive intraepithelial cells, we included labeled folliculotropic and syringotropic lymphocytes, which were shown to have the same diagnostic value as epidermotropic lymphocytes (3). In the dermis, labeled cells were counted in at least four high-power fields (40× objective). We used a computer-assisted method to count labeled dermal cells. First, slides were scanned at high-power $40 \times$ objective. Then, we marked the labeled cells on a superimposed transparency using Adobe Photoshop 6.0 software. Finally, cells were counted on the transparencies using Matrox Inspector 2.2 morphometry software. In the semiquantitative method, slides were independently analyzed by two observers (NO and NB). The infiltrates were then categorized into four groups according to the CD3CD8 ratio: $1(0-25 \%), 2$ (>25-50\%), 3 (>50-75\%), and $4(>75-100 \%)$.

\section{T Cell Clonality}

$\mathrm{T}$ cell clonality was analyzed by deoxyribonucleic acid (DNA) amplification of the T-cell receptor- $\gamma$ gene using consensus primers and separation of the amplimers on denaturing sequencing gel (polymerase chain reactiondenaturing gradient-gel electrophoresis), as described elsewhere $(2,4)$.

\section{Statistical Analysis}

Statistical analyses were conducted using a software package (Statview, Abacus Concepts, Berkeley, CA). Student's $t$ test was used to compare the mean epidermal and dermal CD8-CD3 ratios between mycosis fungoides patients and controls. We also calculated the concordance rates between the quantitative and semiquantitative methods as the percentages of cases whose CD8-CD3 ratios could be categorized in the same group ([0-25\%], [>25$50 \%], \quad[>50-75 \%]$, or $[>75-100 \%])$ using both methods.

\section{RESULTS}

In all mycosis fungoides cases (30/30), a dermal mononuclear cell infiltrate was observed, with epidermotropism present in $27(90 \%)$, in other words, $31(89 \%)$ of the 35 biopsies. In $20(57 \%)$ of the 35 
biopsies, were observed typical mycosis fungoides infiltrates, with bandlike infiltrate and Sézary cells; 19 of them had marked epidermotropism. In the remaining case was found a dense, nonepidermotropic bandlike infiltrate containing Sézary cells, explaining why the lesion was categorized as typical. In 12 (34\%) biopsies, the lesions were suggestive for mycosis fungoides. Three $(9 \%)$ other biopsies showed nonspecific lesions. All samples from mycosis fungoides patients contained CD8+ cells. In all 28 control samples, superficial dermal bandlike or perivascular mononuclear cell infiltrates were observed. Intraepidermal cells were present in 21 of the 28 control specimens (75\%).

The distribution of the cases in the four CD8-CD3 ratio categories $(0-25 \%,>25-50 \%,>50-75 \%,>75-$ $100 \%)$ are shown in Table 1 . The epidermal and dermal CD8-CD3 ratios for each biopsy are shown in Figure 1.

\section{Epidermal Infiltrates}

The quantitative method yielded a mean epidermal CD8-CD3 ratio of 16\% (range: 0-92\%; SD: 25.7) for the mycosis fungoides group, versus $52 \%$ for the controls (range: $4-91 \%$; SD: $28.2 ; P=.0059$ ). Among the 31 biopsies from mycosis fungoides patients displaying epidermotropism, 28 (90\%) had epidermal CD8-CD3 ratios of $<50 \%$, and 25 (81\%) had epidermal ratios of $<25 \%$. CD8-CD3 ratios of $<25 \%$ were obtained for $74 \%$ of the patients with typical mycosis fungoides infiltrates and for $91 \%$ of those with mycosis fungoides-suggestive infiltrates. The sole patient with a nonspecific infiltrate had a CD8CD3 ratio of $<25 \%$. In three biopsies (2 patients, 1 with 2 sequential biopsies), the epidermal CD8CD3 ratio was $>50 \%$ (values: $89 \%, 67 \%$, and $92 \%$ ). When we excluded these two cases from the statistical analysis, the mean epidermal CD8-CD3 ratio for mycosis fungoides patients was $8 \%$ (range: 0-47\%; SD: 12.8 ) and differed significantly from that of the control group $(P<.0001)$. Among the 21 control biopsies with an epidermal component, the CD8-CD3 ratios were $<50 \%$ for $9(43 \%),<25 \%$ for 5
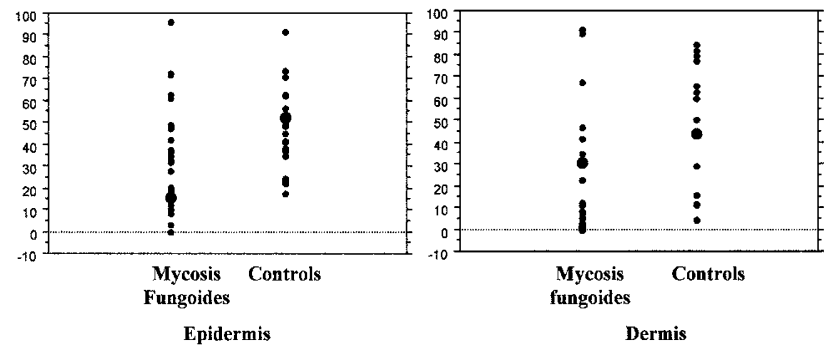

FIGURE 1. CD8-CD3 ratios in the epidermis and in the dermis obtained using the quantitative method. Mean ratios (large point) differed significantly between mycosis fungoides patients and controls, respectively, in the epidermis (15.6 versus $52.2 \% ; P=.0059)$ and the dermis (30.7 versus $44 \% ; P=.0064$ ).

(24\%), and $>75 \%$ for 7 (33\%). Figure 2, A-B shows a mycosis fungoides case classified as suggestive in which the epidermal CD8-CD3 ratio was 0 , and Figure 2, C-D shows a control dermatosis simulating mycosis fungoides with an epidermal ratio of $77 \%$.

\section{Dermal Infiltrates}

The quantitative method gave a mean dermal CD8-CD3 ratio of 31\% (range: $0-96 \%$; SD: 23.6) for mycosis fungoides patients, and $44 \%$ for the controls (range: $17-91 \%$; SD: 18.6; $P=.0064$ ). Twentyseven mycosis fungoides biopsies $(77 \%)$ had dermal CD8-CD3 ratios of $<50 \%$, and 15 (43\%) had ratios of $<25 \%$. Concerning the four mycosis fungoides cases without epidermotropism, three had CD8$\mathrm{CD} 3$ ratios of $<50 \%$, but one had a high CD8-CD3 ratio (96\%). In the control group, 19 biopsies (68\%) had CD8-CD3 ratios of $<50 \%$, and 6 (21\%) had ratios of $<25 \%$. The dermal CD8-CD3 ratio was $37 \%$ in the mycosis fungoides case shown in Figure 2, A-B. Figure 2, C-D shows a control dermatosis with a dermal ratio of $57 \%$.

Concordance rates between the semiquantitative and quantitative methods were higher for epidermal infiltrates $(90 \%$ for mycosis fungoides patients and $95 \%$ for controls) than dermal infiltrates $(54 \%$ for both groups).

TABLE 1. According to the Results Obtained with the Quantitative Method, Distribution of the Cases in the 4 CD8/ CD3 Ratio Categories

\begin{tabular}{|c|c|c|c|c|c|c|c|c|}
\hline \multirow{3}{*}{ Group } & \multicolumn{8}{|c|}{ CD8/CD3 Ratio (\%) } \\
\hline & \multicolumn{4}{|c|}{ Epidermis } & \multicolumn{4}{|c|}{ Dermis } \\
\hline & $0-25$ & $>25-50$ & $>50-75$ & $>75-100$ & $0-25$ & $>25-50$ & $>50-75$ & $75-100$ \\
\hline \multicolumn{9}{|c|}{ Mycosis fungoides } \\
\hline Typical & 74 & 16 & 0 & 10 & 40 & 40 & 20 & 0 \\
\hline Suggestive & 91 & 0 & 9 & 0 & 50 & 25 & 25 & 0 \\
\hline Nonspecific & $100^{\mathrm{a}}$ & 0 & 0 & 0 & 33 & 33 & 0 & 33 \\
\hline Total & 81 & 10 & 3 & 6 & 51 & 26 & 20 & 3 \\
\hline \multicolumn{9}{|l|}{ Controls } \\
\hline Total & 24 & 24 & 19 & 33 & 21 & 46 & 29 & 4 \\
\hline
\end{tabular}

Note that $91 \%$ of the patients with infiltrates suggestive of mycosis fungoides had CD8/CD3 ratios $<25$.

${ }^{a}$ This finding corresponds to only 1 patient and therefore cannot be interpreted. 


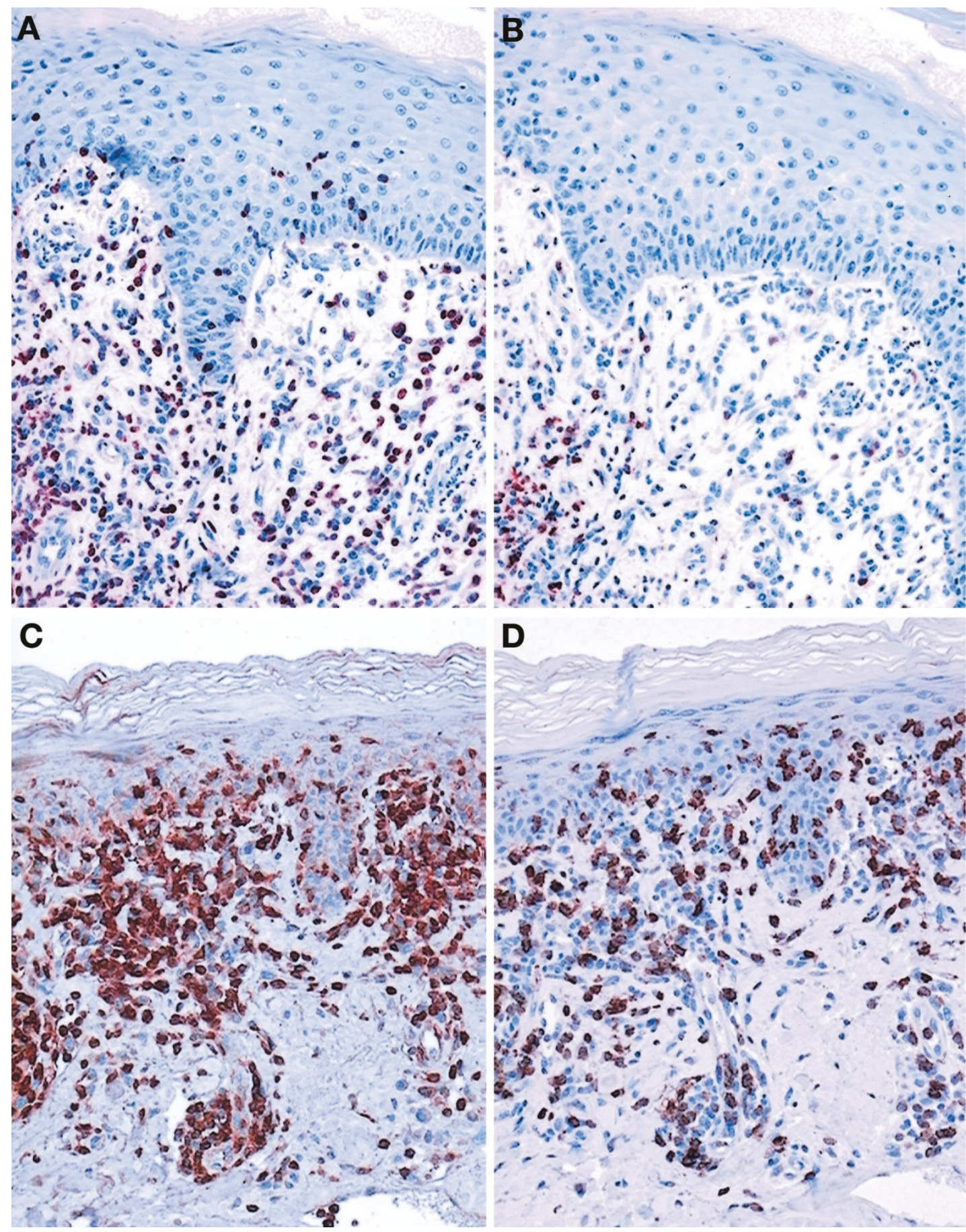

FIGURE 2. A-B, a mycosis fungoides case classified as suggestive morphologically. A, single CD3+ cell epidermotropism is present and numerous CD3+ lymphocytes are observed in the papillary dermis. B, no epidermal lymphocytes express CD8, and only few dermal CD8+ lymphocytes are seen (dermal CD8-CD3 ratio <50\%). C-D, an inflammatory dermatosis simulating mycosis fungoides. C, many CD3+ lymphocytes are present in the epidermis and in the papillary dermis. D, most epidermal lymphocytes express CD8 (CD8-CD3 ratio >75\%), and many CD8+ lymphocytes are present in the dermal infiltrate (CD8-CD3 ratio >50\%).

\section{DISCUSSION}

Histopathological diagnosis of mycosis fungoides is often difficult. Histologically, mycosis fungoides can simulate numerous inflammatory lesions, and histological confirmation of mycosis fungoides is often delayed, compared with clinical suspicion. The most important morphological feature for the diagnosis of mycosis fungoides is the presence of atypical lymphocytes with cerebriform nuclei alone or clustered in the epidermis and in small sheets in the dermis (5). Application use of a standardized method of evaluation of the histopathological criteria could improve the diagnostic accuracy for mycosis fungoides (6). However, the diagnostic value 
of some histological features, especially lymphocytic atypia, is still controversial (7).

Immunohistochemistry has been reported as an additional tool for the diagnosis of mycosis fungoides. Infiltrating lymphocytes in mycosis fungoides are mature CD4 $+\mathrm{T}$ lymphocytes. Thus, it could be expected that immunohistochemical labeling of mycosis fungoides infiltrates would show a dominant CD3+CD4+CD8- population. But the mycosis fungoides infiltrate also comprises nonneoplastic CD8 + T cells. A high CD4-CD8 ratio in the lymphocytic infiltrates of clinically suspicious lesions is, however, considered to be highly suggestive for the histopathological diagnosis of mycosis fungoides. This feature is thought to be highly contributive in early or quiescent mycosis fungoides lesions, which often lack morphological criteria. Actually, few studies have focused on the diagnostic value of the proportion of CD8 + T cells in paraffinembedded mycosis fungoides samples $(8,9)$. To address this issue, we conducted a retrospective immunohistochemical study of 30 cases of early and plaque-stage mycosis fungoides that we compared with 28 control inflammatory dermatoses. CD4 antigen was not assessed because histiocytes, which also express this marker, can be abundant in mycosis fungoides and inflammatory dermatoses. We counted the numbers of cells expressing CD3 and CD8 antigens, expected to be found only on the lymphocytic populations and calculated the CD8CD3 ratios for epidermal and dermal infiltrates. Mycosis fungoides-like infiltration of the skin with numerous CD8+ lymphocytes can be seen in patients with AIDS and may be associated with a poor outcome $(10,11)$. Although true mycosis fungoides can occur in patients with HIV infection, we excluded patients seropositive for HIV to avoid misinterpretation of CD8+ lymphocyte-rich cutaneous infiltrates in this context. In this series, mycosis fungoides diagnosis was reinforced by the presence of a cutaneous dominant clonal T-cell population, in light of the demonstration of their high specificity for mycosis fungoides $(2,12-14)$.

Previous results regarding the diagnostic value of the CD4-CD8 ratio for mycosis fungoides lesions were inconclusive. For example, the percentages of CD8 $+\mathrm{T}$ cells did not differ significantly among mycosis fungoides, non-mycosis fungoides, and uncertain-diagnosis patients (12). In another study, $65 \%$ of the mycosis fungoides lesions had CD4-CD8 ratios of $>2$ (9). According to that study, a high percentage of $\mathrm{CD} 4+$ lymphocytes $(\mathrm{CD} 4-\mathrm{CD} 8$ ratio of $>2$ ) was considered to be specific to the diagnosis of mycosis fungoides. In the present study, the majority of mycosis fungoides lesions $(81 \%)$ had epidermal CD8-CD3 ratios of $<25 \%$, and the mean epidermal CD8-CD3 ratio was significantly lower for mycosis fungoides patients than controls $(P=$
.0059). The difference became more highly significant when we excluded the two mycosis fungoides patients with high epidermal CD8-CD3 ratios $(P<$ $.0001)$, who were retrospectively considered to have $\mathrm{CD} 8+$ mycosis fungoides. Concerning the dermal infiltrates, their CD8-CD3 ratios were also lower for all mycosis fungoides patients than controls, but the statistical difference was slightly lower $(P=$ .0064). Among patients without epidermotropism, three had a low CD8-CD3 ratio $(<50 \%)$, but one had a high CD8-CD3 ratio $(>75 \%)$. Thus a low epidermal CD8-CD3 ratio $(<25 \%)$ is highly suggestive of but not specific to mycosis fungoides. Because only four mycosis fungoides without epidermotropism were included in this series, further studies are needed to determine whether a low dermal CD8CD3 ratio might be useful for diagnosis in such cases. Our results also showed semiquantitative evaluation of the CD8-CD3 ratio to be reliable for epidermal but not dermal infiltrates.

The meaning of the abundant CD8 + T-cell component in the pathogenesis of mycosis fungoides is still unclear. Numerous CD8+ T-cells within mycosis fungoides infiltrate might be a marker of mycosis fungoides with juvenile onset (15). However, this hypothesis remains controversial (16). It has been suggested that CD8+ T-cell-rich mycosis fungoides may have a better prognosis (17). Indeed, CD8 $+\mathrm{T}$ cells in mycosis fungoides infiltrates might be cytotoxic $\mathrm{T}$ lymphocytes directed against tumor cells (18). Furthermore, the difficult distinction must be made between mycosis fungoides with a high proportion of nonclonal CD8+ lymphocytes and CD8+ epidermotropic cutaneous T-cell lymphomas (19).

In conclusion, the presence of a low CD8-CD3 ratio, $<25 \%$, in the epidermal component of a lymphocytic infiltrate is supportive of the diagnosis of mycosis fungoides and can be evaluated in routine practice using a semiquantitative approach. However, this feature is not specific. Because classical lymphocyte phenotyping is not specific for the diagnosis of mycosis fungoides, the search for new specific markers continues.

\section{REFERENCES}

1. Willemze R, Kerl H, Sterry W, Berti E, Cerroni L, Chimenti S, et al. EORTC classification for primary cutaneous lymphomas: a proposal from the Cutaneous Lymphoma Study Group of the European Organization for Research and Treatment of Cancer. Blood 1997;90:354-71.

2. Delfau-Larue MH, Petrella T, Lahet C, Lebozec C, Bagot M, Roudot-Thoraval F, et al. Value of clonality studies of cutaneous $\mathrm{T}$ lymphocytes in the diagnosis and follow-up of patients with mycosis fungoides. J Pathol 1998;184:185-90.

3. Rongioletti F, Smoller B. The histologic value of adnexal (eccrine gland and follicle) infiltration in mycosis fungoides. J Cutan Pathol 2000;27:406-9.

4. Theodorou I, Bigorgne C, Delfau MH, Lahet C, Cochet G, Vidaud M, et al. VJ rearrangements of the TCR gamma locus 
in peripheral T-cell lymphomas: analysis by polymerase chain reaction and denaturing gradient gel electrophoresis. J Pathol 1996;178:303-10.

5. Santucci M, Biggeri A, Feller AC, Massi D, Burg G. Efficacy of histologic criteria for diagnosing early mycosis fungoides: an EORTC cutaneous lymphoma study group investigation. European Organization for Research and Treatment of Cancer Am J Surg Pathol 2000;24:40-50.

6. Guitart J, Kennedy J, Ronan S, Chmiel JS, Hsiegh YC, Variakojis D. Histologic criteria for the diagnosis of mycosis fungoides: proposal for a grading system to standardize pathology reporting. J Cutan Pathol 2001;28:174-83.

7. Yeh YA, Hudson AR, Prieto VG, Shea CR, Smoller BR. Reassessment of lymphocytic atypia in the diagnosis of mycosis fungoides. Mod Pathol 2001;14:285-8.

8. Izban KF, Hsi ED, Alkan S. Immunohistochemical analysis of mycosis fungoides on paraffin-embedded tissue sections. Mod Pathol 1998;11:978-82.

9. Nuckols JD, Shea CR, Horenstein MG, Burchette JL, Prieto VG. Quantitation of intraepidermal T-cell subsets in formalin-fixed, paraffin-embedded tissue helps in the diagnosis of mycosis fungoides. J Cutan Pathol 1999;26:169-75.

10. Guitart J, Variakojis D, Kuzel T, Rosen S. Cutaneous CD8 T cell infiltrates in advanced HIV infection. J Am Acad Dermatol 1999;41:722-7.

11. Zhang P, Chiriboga L, Jacobson M, Marsh E, Hennessey P, Schinella $\mathrm{R}$, et al. Mycosis fungoideslike T-cell cutaneous lymphoid infiltrates in patients with HIV infection. Am J Dermatopathol 1995;17:29-35.

12. Bergman R, Faclieru D, Sahar D, Sander CA, Kerner H, BenAryeh $\mathrm{Y}$, et al. Immunophenotyping and $\mathrm{T}$-cell receptor gamma gene rearrangement analysis as an adjunct to the histopathologic diagnosis of mycosis fungoides. J Am Acad Dermatol 1998;39:554-9.

13. Tok J, Szabolcs MJ, Silvers DN, Zhong J, Matsushima AY. Detection of clonal T-cell receptor gamma chain gene rearrangements by polymerase chain reaction and denaturing gradient gel electrophoresis (PCR/DGGE) in archival specimens from patients with early cutaneous T-cell lymphoma: correlation of histologic findings with PCR/DGGE. J Am Acad Dermatol 1998;38:453-60.

14. Liebmann RD, Anderson B, McCarthy KP, Chow JW. The polymerase chain reaction in the diagnosis of early mycosis fungoides. J Pathol 1997;182:282-7.

15. Whittam LR, Calonje E, Orchard G, Fraser-Andrews EA, Woolford A, Russell-Jones R. CD8-positive juvenile onset mycosis fungoides: an immunohistochemical and genotypic analysis of six cases. Br J Dermatol 2000;143:1199-204.

16. Quaglino P, Zaccagna A, Verrone A, Dardano F, Bernengo MG. Mycosis fungoides in patients under 20 years of age: report of 7 cases, review of the literature and study of the clinical course. Dermatology 1999;199:8-14.

17. Hoppe RT, Medeiros LJ, Warnke RA, Wood GS. CD8-positive tumor-infiltrating lymphocytes influence the long-term survival of patients with mycosis fungoides. J Am Acad Dermatol 1995;32:448-53.

18. Wood GS, Edinger A, Hoppe RT, Warnke RA. Mycosis fungoides skin lesions contain CD8+ tumor-infiltrating lymphocytes expressing an activated, MHC-restricted cytotoxic T-lymphocyte phenotype. J Cutan Pathol 1994;21:151-6.

19. Berti E, Tomasini D, Vermeer MH, Meijer CJ, Alessi E, Willemze R. Primary cutaneous CD8-positive epidermotropic cytotoxic $\mathrm{T}$ cell lymphomas. A distinct clinicopathological entity with an aggressive clinical behavior. Am J Pathol 1999; 155:483-92.

\section{Book Review}

\section{Wikramasinghe SN, McCullough J (eds): Blood and Bone Marrow Pathology, 740 pp, Phil- adelphia, Churchill Livingstone, Elsevier Science, 2003 (\$229.00).}

This multiauthored textbook, edited by a hematologist from London and a transfusiologist from Minneapolis, was written by an international group of some 40 hematologists, hematopathologists, and transfusiologists, most of whom are from the United Kingdom. It covers the pathology of the peripheral blood and the bone marrow and disorders of coagulation. The last 85 pages are devoted to immunohematology and "transfusion medicine for pathologists."

The book, published on high-quality paper and in a large format, contains numerous color illustrations and is physically attractive. The quality of text varies from one chapter to another, reflecting the attempts of various contributors to 'strike a balance between biochemistry, pathophysiology, cytology, histopathology and clinical aspects' of various diseases. Most of the chapters have a very strong clinical tilt and contain data important for diagnosis of hematologic disorders. Details about the processing of tissues and cells are also useful. One wonders, however, why some diseases are included and others are not, and why so much space is allocated to some rare diseases (e.g., transcobalamine II deficiency, to mention just one) at the expense of some more common ones. Electron microscopy, a technique used less often today than two decades ago, also seems to be overrepresented. Molecular biology probably would have deserved more pages. With a few rare exceptions, most photographs are of highest quality. The references are well chosen and current (mostly up to year 2000).

\section{Marin Nola \\ University of Zagreb School of Medicine Zagreb, Croatia}

